\title{
Stability of a vortex in a small trapped Bose-Einstein condensate
}

\author{
Marion Linn ${ }^{1,2}$ and Alexander L. Fetter ${ }^{1}$ \\ ${ }^{1}$ Department of Physics, Stanford University, Stanford, CA 94305-4060 \\ ${ }^{2}$ Physikalisches Institut, Universität Bonn, Nußallee 12, D-53115 Bonn, Germany
}

(June 24, 2018)

\begin{abstract}
A second-order expansion of the Gross-Pitaevskii equation in the interaction parameter determines the thermodynamic critical angular velocity $\Omega_{c}$ for the creation of a vortex in a small axisymmetric condensate. Similarly, a second-order expansion of the Bogoliubov equations determines the (negative) frequency $\omega_{a}$ of the anomalous mode. Although $\Omega_{c}=-\omega_{a}$ through first order, the second-order contributions ensure that the absolute value $\left|\omega_{a}\right|$ is always smaller than the critical angular velocity $\Omega_{c}$. With increasing external rotation $\Omega$, the dynamical instability of the condensate with a vortex disappears at $\Omega^{*}=\left|\omega_{a}\right|$, whereas the vortex state becomes energetically stable at the larger value $\Omega_{c}$. Both second-order contributions depend explicitly on the axial anisotropy of the trap. The appearance of a local minimum of the free energy for a vortex at the center determines the metastable angular velocity $\Omega_{m}$. A variational calculation yields $\Omega_{m}=\left|\omega_{a}\right|$ to first order (hence $\Omega_{m}$ also coincides with the critical angular velocity $\Omega_{c}$ to this order). Qualitatively, the scenario for the onset of stability in the weak-coupling limit is the same as that found in the strong-coupling (Thomas-Fermi) limit.
\end{abstract}

PACS numbers: 03.75.F, 05.30.Jp, 32.80.Pj, 67.40.Db

\section{INTRODUCTION}

The experimental realization of Bose-Einstein condensates [1-3] in dilute alkali gases has stimulated great interest in the study of vortices in these systems. In the absence of experimental detection of a condensate containing a vortex, several authors have addressed the important question of the stability of such a vortex [1 16 . The simplest criterion is energetic stability, determined by comparing the total energy of a condensate with and without a vortex, based on the time-independent GrossPitaevskii equation. Separately, numerical and analytical studies of the Bogoliubov equations for the excitations of a condensate with a vortex found a normal mode with negative energy and positive normalization, suggesting a dynamical instability [7 9, 13]. Moreover, in the limit of large particle number, there is a metastable regime, for which the free energy of the vortex condensate develops a local minimum at the center of the trap, stabilizing the vortex against small lateral displacements [11,15]. In the Thomas-Fermi limit, the onset of metastability coincides with $\left|\omega_{a}\right|$, implying a close connection between the two phenomena [11].

In the present work, we consider a small cylindrically symmetric condensate with a central vortex line along the $z$ axis; this weak-coupling limit allows a perturbation expansion of the Gross-Pitaevskii (GP) equation. The additional energy of the condensate with a vortex can be compensated by rotating the whole condensate with an angular velocity $\Omega_{c}$ [5]. The Bogoliubov equations for the same system have an anomalous mode with negative frequency $\omega_{a}$. To first order in the (small) interaction parameter, $\left|\omega_{a}\right|=\Omega_{c}[9,[16]$. To understand the possible connection between these two types of instabilities, we here evaluate the second-order corrections to
$\Omega_{c}$ and $\omega_{a}$. Furthermore, the metastable frequency $\Omega_{m}$ computed for the weak-coupling limit agrees to first order with the modulus of the anomalous mode, just as in the Thomas-Fermi limit. This result indicates that the instability with respect to microscopic oscillations and the onset metastability are closely related, for the system becomes dynamically stable when rotated faster than the metastable frequency.

The following section uses the GP equation for a condensate with a central vortex to determine the critical rotation frequency $\Omega_{c}$ to second order. In the third section, a similar perturbation expansion of the Bogoliubov equations for the anomalous mode yields the second-order correction to the corresponding eigenvalue of this mode $\omega_{a}$. The fourth section is devoted to determining the metastable frequency $\Omega_{m}$ variationally, and the conclusion discusses the implications of our results.

\section{CRITICAL ANGULAR VELOCITY}

Consider a condensate containing $N$ particles in a harmonic trap with radial and axial frequencies $\omega_{\perp}$ and $\omega_{z}$. The interparticle interactions are characterized by a positive $s$-wave scattering length $a>0$. The starting point for the perturbation theory is the time-independent Gross-Pitaevskii equation [17, 18]

$$
\left(H_{0}+4 \pi \gamma|\Psi|^{2}\right) \Psi=\mu \Psi
$$

with $\mu$ the chemical potential. Here,

$$
H_{0}=\frac{1}{2}\left[-\nabla_{\perp}^{2}+r^{2}+\lambda\left(-\frac{\partial^{2}}{\partial z^{2}}+z^{2}\right)\right]
$$

is the Hamiltonian for the noninteracting condensate, expressed in dimensionless units (the radial and axial 
coordinates are scaled with the radial and axial oscillator lengths $d_{\perp}=\sqrt{\hbar / m \omega_{\perp}}$ and $d_{z}=\sqrt{\hbar / m \omega_{z}}$, and frequencies are scaled with the radial trap frequency $\left.\hbar \omega_{\perp}\right)$. The condensate wave function $\Psi$ is normalized to unity, $\gamma \equiv N a / d_{z}$ is the small interaction parameter and $\lambda=\omega_{z} / \omega_{\perp}$ represents the geometrical asymmetry of the cylindrical trap.

The normalized eigenfunctions for the noninteracting condensate are taken as a product of the one-dimensional axial oscillator state $\varphi_{l}(z)$ and the two-dimensional wave function for a $q$-fold quantized vortex $\chi_{n+q, n}(r, \phi)$ at the center of the trap, so that [compare Eqs. (A1) and (A5) in the appendix]

$$
\psi_{n+q, n, l}(\vec{r})=\chi_{n+q, n}(r, \phi) \varphi_{l}(z)
$$

where $\psi_{q 00}$ is the lowest energy state for a condensate with a given quantized circulation $q$. The noninteracting single-particle states have an energy $\epsilon_{n+q, n, l}^{(0)}=2 n+q+$ $1+\left(l+\frac{1}{2}\right) \lambda$.

The thermodynamic criterion for stability of a rotating condensate with a $q$-fold vortex is the vanishing of the free-energy difference $\Delta F_{q}$ between the free energy of the condensate with and without the vortex. The free energy for a $q$-fold vortex state in the frame rotating with angular velocity $\Omega$ is $F_{q}=E_{q}-\Omega q N$, where $E_{q}$ is the energy of the condensate and $q$ is the angular-momentum quantum number. Setting $F_{q}-F_{0}=0$ gives the critical rotation frequency

$$
\Omega_{c}=\frac{E_{q}-E_{0}}{N q} .
$$

To construct the perturbation theory, the chemical potential and the condensate wave function are expanded in the interaction parameter $\mu \approx \mu^{(0)}+\gamma \mu^{(1)}+\cdots$ and $\Psi \approx \Psi^{(0)}+\gamma \Psi^{(1)}+\cdots$. The first-order correction to the chemical potential of the condensate is easily seen to be $\mu^{(1)}=4 \pi\left\langle\left.\Psi^{(0)}|| \Psi^{(0)}\right|^{2} \mid \Psi^{(0)}\right\rangle$, and the thermodynamic relation $\mu=\partial E / \partial N$ then gives the first-order correction to the condensate energy (note that $\gamma \propto N$ )

$$
E^{(1)}=\frac{1}{2} N \mu^{(1)}=2 \pi N\left\langle\left.\Psi^{(0)}|| \Psi^{(0)}\right|^{2} \mid \Psi^{(0)}\right\rangle .
$$

For a $q$-fold vortex with $\Psi^{(0)}=\psi_{q 00}$, direct evaluation of the matrix element gives

$$
\frac{E_{q}^{(1)}}{N}=\frac{1}{2} \mu_{q}^{(1)}=\frac{(2 q) !}{(q !)^{2} 2^{2 q} \sqrt{2 \pi}} .
$$

The first-order correction to the energy decreases with increasing $q$, and the corresponding critical rotation frequency

$$
\Omega_{c} \approx 1-\frac{E_{0}^{(1)}-E_{q}^{(1)}}{N q} \gamma
$$

increases with increasing $q$. Figure 1 shows the first-order approximation to the thermodynamic critical angular velocity as a function of the interaction parameter $\gamma$ for the first few values of $q$. Since the resulting $\Omega_{c}$ is always smallest for $q=1$, a singly quantized vortex will appear first, in which case $\Omega_{c} \approx 1-\frac{1}{2} \gamma / \sqrt{2 \pi}$. We consider only this case $(q=1)$ in determining the second-order correction to the critical angular velocity.

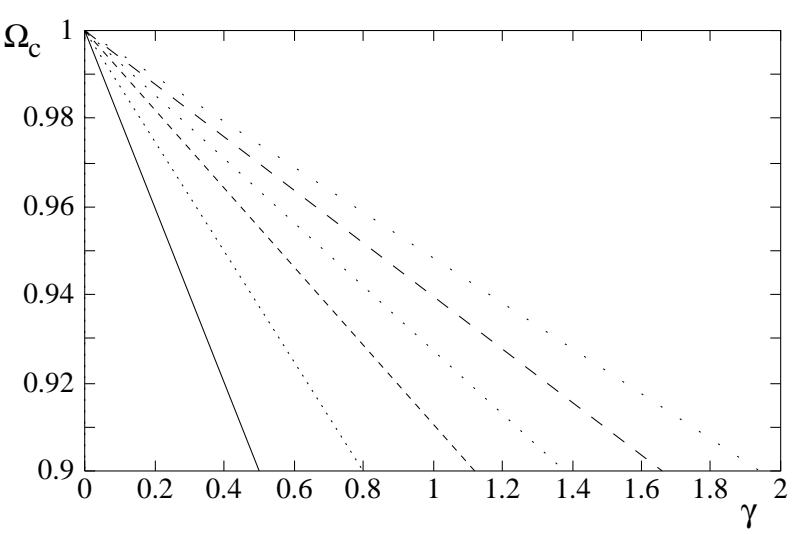

FIG. 1. The critical rotation frequency in first-order perturbation theory for increasing quanta of circulation $q=1 \ldots 6$ (from left to right). The range of the interaction parameter $\gamma$ is extended to unphysical values for better illustration.

The second-order correction to the energy of the condensate can be found by writing $\Psi^{(1)}$ as a sum over the unperturbed eigenfunctions of the appropriate symmetry (this form maintains the normalization to first order)

$$
\Psi^{(1)}=\sum_{n l}^{\prime} c_{n l} \chi_{n+q, n} \varphi_{2 l},
$$

where the prime on the sum indicates that it runs over all nonnegative values of $\{n, l\}$, omitting the single state $\{0,0\}$. Substitution into the GP equation and use of the orthogonality of the unperturbed solutions determine the coefficients

$$
\begin{aligned}
c_{n l} & =-\frac{2 \pi}{n+\lambda l}\left\langle\left.\psi_{n+q, n, 2 l}|| \psi_{q 00}\right|^{2} \mid \psi_{q 00}\right\rangle \\
& =-\frac{2 \pi}{n+\lambda l} I_{l} J_{q q q}^{n q}
\end{aligned}
$$

in terms of definite integrals discussed in the appendix. For example, the axial integration over the product of four harmonic-oscillator eigenfunctions yields

$$
I_{l}=\int_{-\infty}^{\infty} d z \varphi_{2 l}(z) \varphi_{0}(z)^{3}=\frac{(-1)^{l}}{l ! 2^{2 l}} \sqrt{\frac{(2 l) !}{2 \pi}},
$$

with a similar but more complicated expression $J_{i j k}^{n q}$ for the integral over the radial eigenfunctions (they involve Laguerre polynomials).

The corresponding second-order correction to the condensate energy follows immediately from the secondorder chemical potential 


$$
\begin{aligned}
\frac{E_{q}^{(2)}}{N} & =\frac{1}{3} \mu_{q}^{(2)}=16 \pi^{2} \sum_{n l}^{\prime} \frac{\left|\left\langle\left.\psi_{n+q, n, 2 l}|| \psi_{q 00}\right|^{2} \mid \psi_{q 00}\right\rangle\right|^{2}}{\mu_{q}^{(0)}-\epsilon_{n+q, n, 2 l}^{(0)}} \\
& =-8 \pi^{2} \sum_{n l}^{\prime} \frac{\left(I_{l} J_{q q q}^{n, q}\right)^{2}}{n+\lambda l}
\end{aligned}
$$

where $\mu_{q}^{(0)}=\epsilon_{q 00}^{(0)}=q+1+\frac{1}{2} \lambda$. The angular quantum number $q$ is one for the singly quantized vortex or zero for the nonvortex ground state.

In contrast to the first-order correction $E_{q}^{(1)}$ in Eq. (5), the second-order correction now depends explicitly on the asymmetry parameter $\lambda$. The appendix describes a systematic way of writing the matrix elements needed to carry out the infinite sums, and the difference between the second-order energy contributions for the vortex and nonvortex state $\Delta E^{(2)}$ yields the second-order correction to the critical rotation frequency

$$
\Omega_{c}^{(2)}(\lambda)=\frac{1}{16 \pi} \sum_{n l}^{\prime} \frac{1}{n+\lambda l} \frac{12+3 n^{2}-n^{3}}{2^{2 n}} \frac{(2 l) !}{2^{4 l}(l !)^{2}} .
$$

Consequently the second-order approximation to the thermodynamic critical rotation frequency (determined from the GP equation) is

$$
\Omega_{c}=1-\frac{1}{2 \sqrt{2 \pi}} \gamma+\Omega_{c}^{(2)}(\lambda) \gamma^{2}+\cdots
$$

Table I lists the second-order contribution $\Omega_{c}^{(2)}(\lambda)$ for various asymmetry parameters $\lambda$. This contribution is always positive, which counteracts the (negative) firstorder contribution. For large $\lambda$ (a disk-shaped condensate), $\Omega_{c}^{(2)}$ becomes constant (from the terms with $l=0$ ), whereas for small $\lambda$ (a cigar-shaped condensate), it grows like $\Omega_{c}^{(2)} \propto 1 / \lambda$ (from the terms with $n=0$ ). The resulting thermodynamic critical angular velocity for several $\lambda$ is shown in Fig. 2, illustrating the increasing importance of the second-order term for small $\lambda$. For comparison, this figure also includes the critical angular velocity for a $q=1$ vortex with only the first-order correction taken into account.

\begin{tabular}{ccc}
\hline \hline$\lambda$ & $\Omega_{c}^{(2)}$ & $-\omega_{a}^{(2)}$ \\
\hline $10^{4}$ & 0.0805 & 0.0508 \\
10 & 0.0849 & 0.0546 \\
$2 \sqrt{2}$ & 0.0954 & 0.0637 \\
1 & 0.1196 & 0.0851 \\
$10^{-1}$ & 0.4227 & 0.3614 \\
$10^{-2}$ & 3.4033 & 3.0933 \\
$10^{-4}$ & 331.15 & 303.53 \\
\hline \hline
\end{tabular}

TABLE I. Second-order contributions for the critical rotation frequency $\Omega_{c}^{(2)}$ and the anomalous Bogoliubov mode $\omega_{a}^{(2)}$.

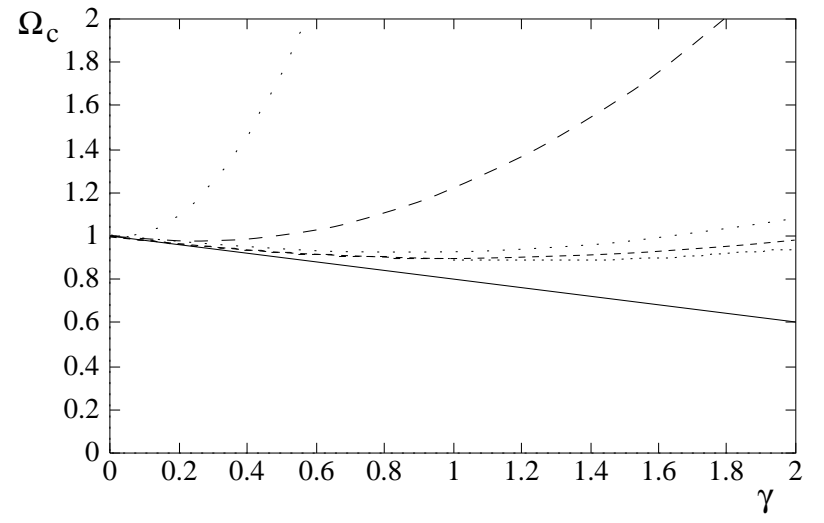

FIG. 2. The critical rotation frequency in second-order perturbation theory for decreasing asymmetry parameters $\lambda=10,2 \sqrt{2}, 1,0.1,0.01$ (from bottom to top). The solid line is $\Omega_{c}$ up to first order. Again, $\gamma$ is extended beyond the range of validity for illustration.

\section{ANOMALOUS MODE}

The preceding section dealt solely with the GP equation for the condensate wave function $\Psi$ and the chemical potential $\mu$, comparing the energy for a condensate with and without a vortex. We now turn to the Bogoliubov equations [19] that describe the small-amplitude excitations of a condensate containing a singly quantized vortex:

$$
\begin{aligned}
\left(H_{0}-\mu+8 \pi \gamma|\Psi|^{2}\right) u_{j}-4 \pi \gamma(\Psi)^{2} v_{j} & =\omega_{j} u_{j}, \\
-4 \pi \gamma\left(\Psi^{*}\right)^{2} u_{j}+\left(H_{0}-\mu+8 \pi \gamma|\Psi|^{2}\right) v_{j} & =-\omega_{j} v_{j},
\end{aligned}
$$

where $u_{j}$ and $v_{j}$ are the normal-mode amplitudes and $\omega_{j}$ is the corresponding frequency. In zero order, the condensate wave function $\Psi^{(0)}$ for a singly quantized vortex is given by $\psi_{100}=\chi_{10} \varphi_{0}$ and the associated chemical potential is $\mu^{(0)}=2+\frac{1}{2} \lambda$. For physical solutions, the normal-mode amplitudes have positive normalization $\int d V\left(\left|u_{j}\right|^{2}-\left|v_{j}\right|^{2}\right)=1$. Here, we focus on the anomalous mode, which has a negative eigenvalue $\omega_{a}<0$. Hence the creation of quasiparticles in this mode lowers the energy relative to that of the condensate, implying a possible instability [7, 9, 13,20].

A noninteracting condensate with a singly quantized vortex is unstable because the particles in the condensate with $\psi_{100}$ can make a transition to the nonrotating condensate with $\psi_{000}$, giving up an energy 1 in our dimensionless units. In the presence of interactions, however, the description becomes more complicated, and the eigenfunctions of the Bogoliubov equation include both $u_{a}$ and $v_{a}$, even in zeroth order. At this level, the Bogoliubov equations for the anomalous mode become

$$
\begin{aligned}
& \left(H_{0}-\mu^{(0)}\right) u_{a}^{(0)}=\omega_{a}^{(0)} u_{a}^{(0)}, \\
& \left(H_{0}-\mu^{(0)}\right) v_{a}^{(0)}=-\omega_{a}^{(0)} v_{a}^{(0)},
\end{aligned}
$$


where the preceding argument suggests that $u_{a}^{(0)} \propto$ $\psi_{000}=\chi_{00} \varphi_{0}$ characterizes the nonrotating vortex-free condensate, so that $\omega_{a}^{(0)}=-1$. Since the full Bogoliubov equation for $u_{a}$ contains the coupling term $(\Psi)^{2} v_{a} \propto$ $e^{2 i \phi} v_{a}$, it is natural to assume that $v_{a} \propto e^{-2 i \phi}$, and the unperturbed state $v_{a}^{(0)} \propto \psi_{020}=\chi_{02} \varphi_{0}$ has the correct energy to satisfy the remaining zero-order Bogoliubov equation. To ensure the proper normalization, we merely take

$$
u_{a}^{(0)}=\cosh \theta \chi_{00} \varphi_{0} \text { and } v_{a}^{(0)}=\sinh \theta \chi_{02} \varphi_{0},
$$

where the parameter $\theta$ can only be determined by including the higher-order terms in the perturbation expansion.

The first-order terms in the Bogoliubov equations become

$$
\begin{aligned}
& \left(H_{0}-\mu^{(0)}\right) u_{a}^{(1)}+\left(8 \pi\left|\Psi^{(0)}\right|^{2}-\mu^{(1)}\right) u_{a}^{(0)} \\
& -4 \pi\left(\Psi^{(0)}\right)^{2} v_{a}^{(0)}=\omega_{a}^{(0)} u_{a}^{(1)}+\omega_{a}^{(1)} u_{a}^{(0)} \\
& \left(H_{0}-\mu^{(0)}\right) v_{a}^{(1)}+\left(8 \pi\left|\Psi^{(0)}\right|^{2}-\mu^{(1)}\right) v_{a}^{(0)} \\
& -4 \pi\left(\Psi^{(0) *}\right)^{2} u_{a}^{(0)}=-\omega_{a}^{(0)} v_{a}^{(1)}-\omega_{a}^{(1)} v_{a}^{(0)},
\end{aligned}
$$

and we must also expand the parameter $\theta \approx \theta^{(0)}+\gamma \theta^{(1)}+$ .... As a result, the zero-order functions in Eq. (18) are evaluated with $\theta^{(0)}$. The first-order contributions then become

$$
\begin{aligned}
& u_{a}^{(1)}=\theta^{(1)} \sinh \theta^{(0)} \chi_{00} \varphi_{0}+\sum_{n l}^{\prime} a_{n l} \chi_{n n} \varphi_{2 l}, \\
& v_{a}^{(1)}=\theta^{(1)} \cosh \theta^{(0)} \chi_{02} \varphi_{0}+\sum_{n l}^{\prime} b_{n l} \chi_{n, n+2} \varphi_{2 l} ;
\end{aligned}
$$

these expansions maintain the proper normalization to first order in $\gamma$.

Multiply Eqs. (19) and (20) by $u_{a}^{(0) *}$ and $v_{a}^{(0) *}$, respectively, and integrate. Straightforward manipulations yield the pair of equations

$$
\begin{aligned}
\mu^{(1)}+\omega_{a}^{(1)}= & 4 \pi I_{0}\left(2 \int d^{2} r\left|\chi_{00}\right|^{2}\left|\chi_{10}\right|^{2}\right. \\
& \left.-\tanh \theta^{(0)} \int d^{2} r \chi_{00}^{*}\left(\chi_{10}\right)^{2} \chi_{02}\right) \\
= & \frac{1}{\sqrt{2 \pi}}\left(2-\frac{\tanh \theta^{(0)}}{\sqrt{2}}\right), \\
\mu^{(1)}-\omega_{a}^{(1)}= & 4 \pi I_{0}\left(2 \int d^{2} r\left|\chi_{02}\right|^{2}\left|\chi_{10}\right|^{2}\right. \\
& \left.-\operatorname{coth} \theta^{(0)} \int d^{2} r \chi_{02}^{*}\left(\chi_{10}^{*}\right)^{2} \chi_{00}\right) \\
= & \frac{1}{\sqrt{2 \pi}}\left(\frac{3}{2}-\frac{\operatorname{coth} \theta^{(0)}}{\sqrt{2}}\right),
\end{aligned}
$$

where $I_{0}$ is given in Eq. (10), and the radial integrals are evaluated with the results in the appendix. The sum of these equations determines $\mu^{(1)}$, and comparison with Eq. (6) yields

$$
\begin{aligned}
& \cosh \theta^{(0)}=\sqrt{2}, \quad \text { and } \\
& \sinh \theta^{(0)}=1 .
\end{aligned}
$$

Correspondingly, the difference now determines the firstorder correction to the anomalous frequency [9]

$$
\omega_{a}^{(1)}=\frac{1}{2 \sqrt{2 \pi}}
$$

so that $\Omega_{c}=-\omega_{a}$ through first order.

To determine the coefficients in the first-order expansions in Eqs. 21) and (22), project Eqs. (19) and (20) onto the appropriate unperturbed eigenfunctions. In this way, we find (see Appendix B)

$$
\begin{aligned}
a_{n l}= & \frac{2 \pi I_{l}}{n+\lambda l}\left(\int d^{2} r \chi_{n n}^{*}\left(\chi_{10}\right)^{2} \chi_{02}\right. \\
& \left.-2 \sqrt{2} \int d^{2} r \chi_{n n}^{*}\left|\chi_{10}\right|^{2} \chi_{00}\right) \\
= & \frac{2 \pi I_{l}}{n+\lambda l}\left(J_{112}^{n 0}-2 \sqrt{2} J_{110}^{n 0}\right), \\
b_{n l}= & \frac{2 \pi I_{l}}{n+\lambda l}\left(\sqrt{2} \int d^{2} r \chi_{n, n+2}^{*}\left|\chi_{10}\right|^{2} \chi_{02}\right. \\
& \left.-2 \int d^{2} r \chi_{n, n+2}^{*}\left(\chi_{10}^{*}\right)^{2} \chi_{00}\right) \\
= & \frac{2 \pi I_{l}}{n+\lambda l}\left(\sqrt{2} J_{112}^{n 2}-2 J_{110}^{n 2}\right) .
\end{aligned}
$$

The second-order Bogoliubov equations are

$$
\begin{aligned}
& \left(\omega_{a}^{(0)} u_{a}^{(2)}+\omega_{a}^{(1)} u_{a}^{(1)}+\omega_{a}^{(2)} u_{a}^{(0)}\right) \\
& =\left(H_{0}-\mu^{(0)}\right) u_{a}^{(2)}-\mu^{(1)} u_{a}^{(1)}-\mu^{(2)} u_{a}^{(0)} \\
& +8 \pi\left[\left|\Psi^{(0)}\right|^{2} u_{a}^{(1)}+\left(\Psi^{(0) *} \Psi^{(1)}+\Psi^{(0)} \Psi^{(1) *}\right) u_{a}^{(0)}\right] \\
& -4 \pi\left[\left(\Psi^{(0)}\right)^{2} v_{a}^{(1)}+2 \Psi^{(0)} \Psi^{(1)} v_{a}^{(0)}\right] \\
& -\left(\omega_{a}^{(0)} v_{a}^{(2)}+\omega_{a}^{(1)} v_{a}^{(1)}+\omega_{a}^{(2)} v_{a}^{(0)}\right) \\
& =\left(H_{0}-\mu^{(0)}\right) v_{a}^{(2)}-\mu^{(1)} v_{a}^{(1)}-\mu^{(2)} v_{a}^{(0)} \\
& +8 \pi\left[\left|\Psi^{(0)}\right|^{2} v_{a}^{(1)}+\left(\Psi^{(0) *} \Psi^{(1)}+\Psi^{(0)} \Psi^{(1) *}\right) v_{a}^{(0)}\right] \\
& -4 \pi\left[\left(\Psi^{(0) *}\right)^{2} u_{a}^{(1)}+2 \Psi^{(0) *} \Psi^{(1) *} u_{a}^{(0)}\right]
\end{aligned}
$$

Here, Eqs. (8) and (9) give the condensate wave function $\Psi^{(1)}$, and Eqs. (21), (22), (27) and (28) give the corrections $u_{a}^{(1)}$ and $v_{a}^{(1)}$.

The remaining steps are essentially the same as in first order, yielding a pair of equations for $\mu^{(2)} \pm \omega_{a}^{(2)}$. The parameter $\theta^{(1)}$ must be chosen so that the sum reproduces Eq. (11) for the chemical potential $\mu^{(2)}$. The difference then gives the second-order correction to the anomalous frequency 


$$
\begin{aligned}
\omega_{a}^{(2)}(\lambda)= & 8 \pi^{2} \sum_{n l}^{\prime} \frac{\left(I_{l}\right)^{2}}{n+l \lambda} \\
\times & {\left[13\left(J_{111}^{n 1}\right)^{2}-8\left(J_{110}^{n 0}\right)^{2}-4\left(J_{112}^{n 2}\right)^{2}-2\left(J_{110}^{n 2}\right)^{2}\right.} \\
& -\left(J_{112}^{n 0}\right)^{2}-8 J_{100}^{n 1} J_{111}^{n 1}-4 J_{122}^{n 1} J_{111}^{n 1} \\
& \left.+4 \sqrt{2}\left(J_{112}^{n 0} J_{110}^{n 0}+J_{110}^{n 2} J_{112}^{n 2}\right)\right]
\end{aligned}
$$

and the total anomalous frequency through second order becomes

$$
\omega_{a}=-1+\frac{1}{2} \frac{1}{\sqrt{2 \pi}} \gamma+\omega_{a}^{(2)}(\lambda) \gamma^{2} .
$$

This is the main result of the present section, illustrated in Fig. 3.

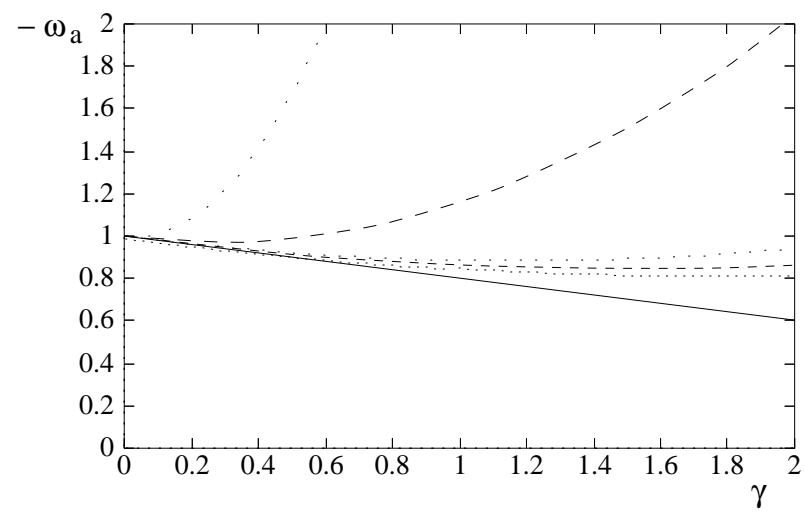

FIG. 3. The absolute values of the anomalous mode frequency in second-order perturbation theory for decreasing asymmetry parameters $\lambda=10,2 \sqrt{2}, 1,0.1,0.01$ (from bottom to top). The solid line is $\omega_{a}$ up to first order.

For several different axial asymmetries, Table I compares the second-order correction $\Omega_{c}^{(2)}(\lambda)$ to the critical rotation frequency from the preceding section with the second-order correction $\omega_{a}^{(2)}(\lambda)$ to the anomalous frequency. It can be seen that $\omega_{a}^{(2)}$ is always negative and smaller in absolute value than the corresponding correction $\Omega_{c}^{(2)}$.

To understand the significance of this result, consider a normal mode of the $q$-fold quantized vortex with $u_{j}(r, \phi)=\exp \left[i\left(m_{j}+q\right) \phi\right] \tilde{u}_{j}(r)$ and $v_{j}(r, \phi)=\exp \left[i\left(m_{j}-\right.\right.$ q) $\phi] \tilde{v}_{j}(r)$ [7, 10], where $m_{j}$ is the angular momentum of the normal mode relative to that of the vortex in the condensate (namely, the corresponding perturbations in the density and velocity potential have the angular dependence $\propto \exp i m_{j} \phi$ ). In a frame rotating with angular velocity $\Omega$, the frequency of a given normal mode becomes $\omega_{j}(\Omega)=\omega_{j}(0)-m_{j} \Omega$. For the present case of a singly quantized vortex with $q=1$, the anomalous mode has $m_{a}=-1$, so that the shifted anomalous frequency in the rotating frame is $\omega_{a}(\Omega)=\omega_{a}(0)+\Omega$. With increasing external rotation $\Omega$, the condensate with a vortex becomes dynamically stable against microscopic oscillations for $\Omega \geq \Omega^{*}=\left|\omega_{a}\right|$. Our numerical results show that $\Omega^{*}<\Omega_{c}$, so the onset of dynamical stability occurs before the singly quantized vortex in the condensate becomes energetically favorable. Since the sum $\Omega_{c}+\omega_{a}$ vanishes through order $\gamma$, it is necessary to include the second-order corrections to decide the relative value of $\Omega^{*}$ and $\Omega_{c}$. The difference is illustrated in Fig. 4 for two geometries, including the corresponding results for strong coupling (in the Thomas-Fermi limit) [11], which shows that the sequence of the two types of stabilization is the same in both regimes.

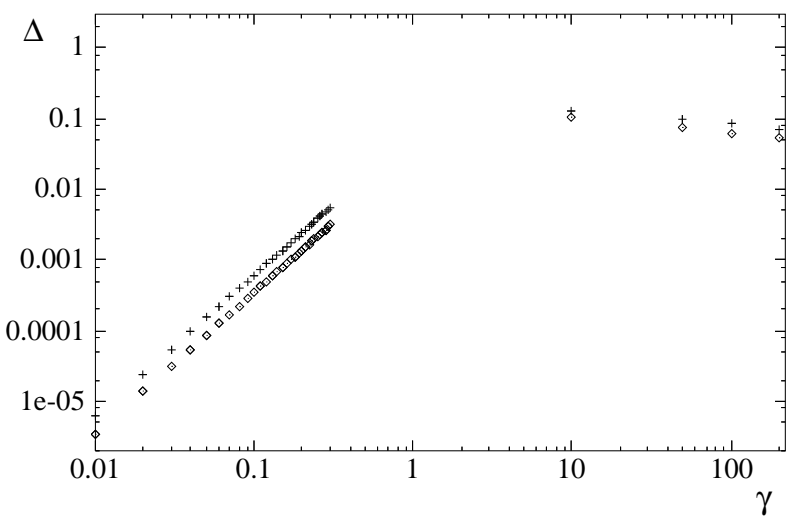

FIG. 4. The difference $\Delta=\Omega_{c}-\left|\omega_{a}\right|$ (namely $\Omega_{c}+\omega_{a}$ ) for the asymmetries $\lambda=1$ (diamonds) and $\lambda=0.1$ (crosses). In the weak-coupling limit, the difference is much smaller than in the Thomas-Fermi limit (note the logarithmic scale), but remains positive.

\section{METASTABLE FREQUENCY}

The third important frequency in this problem is the metastable angular velocity $\Omega_{m}$ at which a slightly offcenter vortex becomes trapped in a local minimum of the free energy at the center of the confining potential. Consider the corresponding energy functional

$$
E(\Psi)=\int d V\left[\Psi^{*}\left(H_{0}-\Omega L_{z}\right) \Psi+2 \pi \gamma|\Psi|^{4}\right] .
$$

We now use cartesian coordinates, with

$$
H_{0}=\frac{1}{2}\left[-\partial_{x}^{2}-\partial_{y}^{2}+\left(x^{2}+y^{2}\right)+\lambda\left(-\partial_{z}^{2}+z^{2}\right)\right]
$$

and $L_{z}=-i(x \partial y-y \partial x)$ is the $z$-component of the angular-momentum operator.

In order to evaluate the energy functional Eq. (33), we construct a trial condensate wave function. This trial function is assumed to be unchanged along the axis of 
symmetry, which allows us to use the ground-state gaus$\operatorname{sian} \varphi_{0}(z)$. In the weak-coupling limit, the radius of the vortex core is comparable with the radius of the condensate, so that the displacement $\mathbf{r}_{0}=\left(x_{0}, y_{0}\right)$ of the vortex, the displacement $\mathbf{r}_{1}=\left(x_{1}, y_{1}\right)$ of the condensate, and the induced velocity of the condensate must all be included in the following trial function

$$
\begin{aligned}
\psi_{v}(x, y, z) & =\frac{C}{\pi^{3 / 4}}\left[\left(x-x_{0}\right)+i\left(y-y_{0}\right)\right] \\
& \times e^{-\frac{1}{2}\left(x-x_{1}\right)^{2}-\frac{1}{2}\left(y-y_{1}\right)^{2}-\frac{1}{2} z^{2}} e^{i\left(\alpha_{x} x+\alpha_{y} y\right)},
\end{aligned}
$$

where $C^{-2}=1+\left|\mathbf{r}_{1}-\mathbf{r}_{0}\right|^{2}$ and the constants $\alpha_{x}$ and $\alpha_{y}$ characterize the velocity components.

In evaluating the integration in $E\left(\psi_{v}\right)$, we retain all terms up to second order in the small parameters $\alpha_{i}$ and in the displacements. For convenience, we introduce new variables $\boldsymbol{\delta}=\mathbf{r}_{1}-\mathbf{r}_{0}=\left(\delta_{x}, \delta_{y}\right)$ and $\boldsymbol{\epsilon}=2 \mathbf{r}_{1}-\mathbf{r}_{0}=$ $\left(\epsilon_{x}, \epsilon_{y}\right)$, which will turn out to be the normal modes of the system. The variational energy is

$$
\begin{aligned}
E_{\mathrm{var}}(\boldsymbol{\alpha}, \boldsymbol{\delta}, \boldsymbol{\epsilon}) & =2+\frac{\lambda}{2}-\Omega+\frac{\gamma}{2 \sqrt{2 \pi}} \\
& +\frac{\alpha^{2}}{2}+\alpha_{x}\left(-\delta_{y}+\Omega \epsilon_{y}\right)+\alpha_{y}\left(\delta_{x}-\Omega \epsilon_{x}\right) \\
& +\frac{\epsilon^{2}}{2}-\Omega \boldsymbol{\delta} \cdot \boldsymbol{\epsilon}+\left(2 \Omega-\frac{3}{2}+\frac{\gamma}{\sqrt{2 \pi}}\right) \delta^{2}
\end{aligned}
$$

The values

$$
\begin{aligned}
& \alpha_{x}=\delta_{y}-\Omega \epsilon_{y} \\
& \alpha_{y}=-\delta_{x}+\Omega \epsilon_{x}
\end{aligned}
$$

minimize this expression with respect to the velocity parameter $\boldsymbol{\alpha}=\left(\alpha_{x}, \alpha_{y}\right)$, and the energy then becomes diagonal in the variables $\boldsymbol{\delta}$ and $\boldsymbol{\epsilon}$, which thus represent the appropriate normal modes

$$
\begin{aligned}
E_{\mathrm{var}}(\boldsymbol{\delta}, \boldsymbol{\epsilon}) & =2+\frac{\lambda}{2}+\frac{\gamma}{2 \sqrt{2 \pi}}-\Omega \\
& +\left(-2+\frac{\gamma}{\sqrt{2 \pi}}+2 \Omega\right) \delta^{2}+\frac{\left(1-\Omega^{2}\right)}{2} \epsilon^{2} .
\end{aligned}
$$

The energy of the vortex-free ground state is $E_{0}=1+$ $\lambda / 2+\gamma /(\sqrt{2 \pi})$. Thus the difference $\Delta F(0)=E_{\mathrm{var}}(0)-E_{0}$ vanishes at the expected critical angular velocity $\Omega_{c}=$ $1-\gamma /(2 \sqrt{2 \pi})$, in agreement with the first-order result obtained from the Gross-Pitaevskii equation. Furthermore, if $\boldsymbol{\delta}$ vanishes, then the vortex and condensate move rigidly, and the resulting dipole oscillation mode is stable throughout the range $|\Omega| \leq 1$. For larger angular velocity, the motion becomes unstable, as is familiar from the behavior of a classical particle in a parabolic potential.

We next consider how the relative displacement $\boldsymbol{\delta}$ affects the variational energy Eq. (39), which is clearly unstable if $\Omega=0$. With increasing external rotation, however, the relative displacement becomes metastable at a frequency

$$
\Omega_{m}=1-\frac{\gamma}{2 \sqrt{2 \pi}},
$$

when $E_{\text {var }}$ changes from a local maximum to a local minimum for small relative displacements. To this (first) order in the interaction parameter $\gamma$, the value $\Omega_{m}$ coincides with the modulus of the anomalous mode frequency $\left|\omega_{a}\right|$ and hence with the critical frequency $\Omega_{c}$.

In order to illustrate the importance of the combined effect of the vortex and the condensate, we can study the behavior if only the vortex is displaced (namely $\mathbf{r}_{1}=0$ ) or if the induced velocity is neglected $(\boldsymbol{\alpha}=0)$. In the former case, the energy (39) is given by

$$
\begin{aligned}
E_{\mathrm{var}}\left(\mathbf{r}_{0}\right) & =2+\frac{\lambda}{2}+\frac{\gamma}{2 \sqrt{2 \pi}}-\Omega \\
& +\left(-\frac{3}{2}+\frac{\gamma}{\sqrt{2 \pi}}+2 \Omega-\frac{\Omega^{2}}{2}\right)\left(x_{0}^{2}+y_{0}^{2}\right),
\end{aligned}
$$

and the metastable frequency is determined by requiring a positive coefficient of the displacement contribution [the second line in Eq. (41)]. To first order in the interaction parameter, we find

$$
\Omega_{m}^{*}=1-\frac{\gamma}{\sqrt{2 \pi}}
$$

which differs from the value $\Omega_{m}$ found with the more general approach. In the second case, the metastable frequency is determined by the condition that the last line in Eq. (36) is positive, namely the determinant of the coefficients must be positive. This gives the same metastable frequency (42) as that found from Eq. (41). Therefore, the metastable frequency has the same value as the absolute value of the anomalous mode (up to first order) only if the displacement of the condensate and the induced velocity are both taken into account. This indicates that the vortex becomes confined in a local central energy minimum at the same rotation frequency $\left|\omega_{a}\right|$ for which the instability due to the anomalous mode disappears. The same scenario had been found in the strongcoupling limit 11, and our results strengthen the idea of a common underlying phenomenon.

Note that the metastable frequency found here [Eq. (40)] does not coincide with the metastable rotation frequency defined by Feder, Clark, and Schneider [15]. Instead of requiring a local minimum in the free energy, they identify the onset of metastability with the frequency for which the chemical potentials for a condensate with and without a vortex are equal. In firstorder perturbation theory, their criterion leads to the expression (42) found by omitting the displacement of the condensate.

\section{CONCLUSIONS}

It has been shown that the critical rotation frequency $\Omega_{c}$ exceeds the modulus of the anomalous Bogoliubov 
mode $\omega_{a}$ when second-order corrections in the interaction parameter are included. This result agrees with numerical results for one particular trap geometry found by Feder, Clark, and Schneider [15]. Furthermore, the sequence of stabilizing a singly quantized vortex through rotation is found to be the same as in the strong-coupling limit 11]: First, the vortex becomes stable against microscopic oscillations at $\Omega^{*}=\left|\omega_{a}\right|$, and only at the higher rotation speed $\Omega_{c}$ does the vortex become energetically stable. The geometry dependence introduced through the second-order terms indicates that pancake geometries are more favorable for vortex detection in rotating traps, since the stabilization frequencies are lower. This is consistent with the numerical results of GarcíaRipoll and Pérez-García [14]. For extreme cigar-shaped condensates, the critical frequency can even exceed the trap frequencies 15], a regime that is experimentally inaccessible. The angular velocity $\Omega_{m}$ for the onset of metastability coincides with the modulus of the anomalous frequency $\omega_{a}$ in first order, when the combined effect of the vortex and the condensate is taken into account. For $\Omega>\Omega_{m}$, an energy barrier stabilizes the vortex at the center; at the same frequency, the instability due to microscopic oscillations disappears. These features in the stability scenario also agree with the behavior in the Thomas-Fermi limit 11].

\section{ACKNOWLEDGMENTS}

We thank A. A. Svidzinsky for valuable discussions. This work was supported in part by NSF Grant No. 94-21888 and by the DAAD (German Academic Exchange Service) "Doktorandenstipendium im Rahmen des gemeinsamen Hochschulsonderprogramms III von Bund und Ländern” (M. L.).

\section{APPENDIX A: NONINTERACTING EIGENSTATES}

The eigenstates for the noninteracting Bose condensate in a cylindrical trap can be classified with the quantum numbers of positive and negative circulation $n_{+}, n_{-}$ around the $z$ axis and the axial harmonic-oscillator energy quantum number $l$ in the $z$ direction [21]. In particular, the $z$-dependent parts of the eigenfunctions are simple harmonic oscillator eigenfunctions

$$
\varphi_{l}(z)=\frac{1}{\sqrt{\pi^{1 / 2} 2^{l} l !}} H_{l}(z) e^{-\frac{1}{2} z^{2}},
$$

where the $H_{l}(z)$ are the Hermite polynomials [22].

In terms of the circular quanta, the normalized twodimensional eigenfunctions are

$\chi_{n_{+}, n_{-}}(x, y)=\frac{1}{\sqrt{\pi n_{+} ! n_{-} !}}\left(a_{+}^{\dagger}\right)^{n_{+}}\left(a_{-}^{\dagger}\right)^{n_{-}} e^{-\frac{1}{2}\left(x^{2}+y^{2}\right)}$, where $a_{ \pm}^{\dagger}=\left(a_{x}^{\dagger} \pm i a_{y}^{\dagger}\right) / \sqrt{2}=\frac{1}{2}\left[x \pm i y-\left(\partial_{x} \pm i \partial_{y}\right)\right]$ are the creation operators for right and left circular quanta, respectively. In terms of the new variables $\zeta=x+i y$, $\zeta^{*}=x-i y$, these operators take the form

$$
a_{+}^{\dagger}=\frac{\zeta}{2}-\frac{\partial}{\partial \zeta^{*}}, \quad a_{-}^{\dagger}=\frac{\zeta^{*}}{2}-\frac{\partial}{\partial \zeta} .
$$

The identity $\left(\frac{1}{2} \zeta^{*}-\partial_{\zeta}\right) \exp \left(-\frac{1}{2} \zeta \zeta^{*}\right)=\exp \left(\frac{1}{2} \zeta \zeta^{*}\right)\left(-\partial_{\zeta}\right)$ $\exp \left(-\zeta \zeta^{*}\right)$ and its complex conjugate readily yield

$$
\begin{aligned}
\chi_{n+q, n} & =\frac{e^{\frac{1}{2} \zeta \zeta^{*}}}{\sqrt{\pi(n+q) ! n !}}\left(-\frac{\partial}{\partial \zeta}\right)^{n}\left(-\frac{\partial}{\partial \zeta^{*}}\right)^{n+q} e^{-\zeta \zeta^{*}} \\
& \left.=\frac{(-1)^{n} e^{\frac{1}{2} \zeta \zeta^{*}}}{\sqrt{\pi(n+q) ! n !}}\left(\frac{\partial}{\partial \zeta}\right)^{n}\left(\zeta^{n+q} e^{-\zeta \zeta^{*}}\right) \cdot \quad \text { (A4 }\right)
\end{aligned}
$$

Comparison with the standard formula for the associated Laguerre polynomials $L_{n}^{q}$ [22] yields

$$
\chi_{n+q, n}(r, \phi)=(-1)^{n} \sqrt{\frac{n !}{\pi(n+q) !}} e^{-\frac{1}{2} r^{2}} e^{i q \phi} r^{q} L_{n}^{q}\left(r^{2}\right) .
$$

The complete normalized three-dimensional eigenfunctions of the noninteracting system are $\psi_{n+q, n, l}(\vec{r})=$ $\chi_{n+q, n}(r, \phi) \varphi_{l}(z)$.

\section{APPENDIX B: SECOND-ORDER MATRIX ELEMENTS}

The above expressions allow us to evaluate the necessary matrix elements, and the $z$-dependent part factorizes out in the form

$$
\begin{aligned}
I_{l} & =\int_{-\infty}^{\infty} d z \varphi_{2 l}(z) \varphi_{0}(z)^{3} \\
& =\frac{1}{\pi \sqrt{2^{2 l}(2 l) !}} \int_{-\infty}^{\infty} d z H_{2 l}(z) e^{-2 z^{2}},
\end{aligned}
$$

where only states with an even number of quanta contribute. The generating function for the Hermite polynomials

$$
e^{-t^{2}+2 z t}=\sum_{n=0}^{\infty} \frac{H_{n}(z)}{n !} t^{n}
$$

readily shows that $\exp \left(-\frac{1}{2} t^{2}\right)$ is the generating function for $\sqrt{2^{2 l}(2 l) !} I_{l}$, and a straightforward analysis gives the desired expression

$$
I_{l}=\frac{1}{\sqrt{2 \pi}} \frac{(-1)^{l} \sqrt{(2 l) !}}{2^{2 l} l !} .
$$

For the radial part note that $\chi_{n, n+q}(r, \phi)=$ $\chi_{n+q, n}^{*}(r, \phi)$. Since the phase factor is the only complex part, it can be separated explicitly 


$$
\begin{aligned}
& \chi_{n+q, n}(r, \phi)=e^{i q \phi} \tilde{\chi}_{n+q, n}(r), \\
& \chi_{n, n+q}(r, \phi)=e^{-i q \phi} \tilde{\chi}_{n+q, n}(r),
\end{aligned}
$$

where $\tilde{\chi}$ is real. The matrix elements $J_{i j k}^{n q}=$ $\int d^{2} r \tilde{\chi}_{n+q, n} \tilde{\chi}_{i, 0} \tilde{\chi}_{j, 0} \tilde{\chi}_{k, 0}$ involve a product of four of these eigenfunctions, where three refer to the condensate in the lowest energy state, namely $n=0$ (note that $L_{0}^{\alpha}=1$ for any $\alpha$ ). Due to the angular integration, only products with no net overall phase remain. Use of Eq. A5) yields

$$
J_{i j k}^{n q}=\frac{(-1)^{n}}{\pi} \frac{1}{\sqrt{i ! j ! k !}} \sqrt{\frac{n !}{(n+q) !}} \int_{0}^{\infty} d u u^{p} e^{-2 u} L_{n}^{q}(u),
$$

where $p=\frac{1}{2}(q+i+j+k)$ is an integer because of the angular phase factors (note also that $J_{i j k}^{n q}$ is symmetric under interchange of its subscripts). As in the preceding example of the axial matrix elements, the generating function for the Laguerre polynomials 22]

$$
\sum_{n=0}^{\infty} L_{n}^{q}(u) t^{n}=\frac{1}{(1-t)^{1+q}} \exp \left(\frac{-u t}{1-t}\right)
$$

facilitates the radial integration. For example the function $2(1-t) /(2-t)^{3}$ provides a generating function for $(-1)^{n} \pi \sqrt{n+1} J_{111}^{n 1}$, which is the matrix element with four singly quantized vortex eigenfunctions, and we find

$$
J_{111}^{n 1}=\frac{(-1)^{n}}{2^{n+3} \pi} \sqrt{n+1}(2-n) .
$$

A similar technique leads to all the other relevant radial integrals.

The sums occurring in the second-order terms contain the square of the matrix elements $\left(J_{i j k}^{n q} I_{l}\right)^{2}$ appropriately weighted with the energy denominator. For example, the second-order contribution to the chemical potential for the singly quantized vortex (obtained from the GrossPitaevskii equation) is

$$
\begin{aligned}
\mu_{1}^{(2)} & =-24 \pi^{2} \sum_{n l}^{\prime} \frac{\left(J_{111}^{n 1} I_{l}\right)^{2}}{n+l \lambda} \\
& =-\frac{3}{16 \pi} \sum_{n l}^{\prime} \frac{1}{n+\lambda l} \frac{(n+1)(2-n)^{2}}{2^{2 n}} \frac{(2 l) !}{2^{4 l}(l !)^{2}}
\end{aligned}
$$

[2] K. B. Davis, M.-O. Mewes, M. R. Andrews, N. J. van Druten, D. S. Durfee, D. M. Kurn, and W. Ketterle, Phys. Rev. Lett. 75, 3969 (1995).

[3] C. C. Bradley, C. A. Sackett, and R. G. Hulet, Phys. Rev. Lett. 78, 985 (1997).

[4] G. Baym and C. J. Pethick, Phys. Rev. Lett. 76, 6 (1996).

[5] F. Dalfovo and S. Stringari, Phys. Rev. A53, 2477 (1996).

[6] E. Lundh, C. J. Pethick, and H. Smith, Phys. Rev. A 55, 2126 (1997).

[7] R. J. Dodd, K. Burnett, M. Edwards, and C. W. Clark, Phys. Rev. A 56, 587 (1997).

[8] D. S. Rokhsar, Phys. Rev. Lett. 79, 2164 (1997).

[9] A. L. Fetter, J. Low Temp. Phys. 113, 198 (1998).

[10] A. A. Svidzinsky and A. L. Fetter, Phys. Rev. A 58, 3168 (1998).

[11] A. A. Svidzinsky and A. L. Fetter, cond-mat/9811348.

[12] H. Pu, C. K. Low, J. H. Eberly, and N. P. Bigelow, Phys. Rev. A. 59, 1533 (1999).

[13] T. Isoshima and K. Machida, J. Phys. Soc. Jpn. 68, 487 (1999).

[14] J. J. García-Ripoll and V. M. Pérez-García, condmat/9903353.

[15] D. L. Feder, C. W. Clark, and B. I. Schneider, condmat/9904269.

[16] D. A. Butts and D. S. Rokhsar, Nature 397, 327 (1999).

[17] L. P. Pitaevskii, Zh. Éksp. Teor. Fiz. 40, 646 (1961) [Sov. Phys. JETP 13, 45 (1961)].

[18] E. P. Gross, Nuovo Cimento 20, 454 (1961); J. Math. Phys. 4, 195 (1963).

[19] N. Bogoliubov, J. Phys. (Moscow) 11, 23 (1947).

[20] A. L. Fetter, in Proceedings of the International School of Physics "Enrico Fermi," July, 1998, to be published.

[21] C. Cohen-Tannoudji, B. Diu, F. Laloë, Quantum Mechanics (Wiley, 1977), Vol. I, pp. 500-502, 733-738.

[22] N. N. Lebedev, Special Functions and Their Applications (Dover, 1972), Chap. 4.
[1] M. H. Anderson, J. R. Ensher, M. R. Matthews, C. E. Wieman, and E. A. Cornell, Science 269, 198 (1995). 